\title{
Avaliação do conhecimento de professores do ensino fundamental da rede particular sobre atendimento imediato de vítima de traumatismo dental
}

\section{Knowledge assessment of elementary school teachers from the private school network on the immediate assistance of dental trauma victims}

Layanne Soyara Bidô Alves* Vicente Jadson Gregório Freitas*

Rosana Araújo Rosendo***

Luciana Ferraz Gominho**+*

Tassia Cristina de Almeida Pinto-Sarmento ${ }^{* * *+*}$

\section{Resumo}

Objetivo: esta pesquisa objetiva reconhecer o nível de conhecimento dos professores do ensino fundamental da rede particular na cidade de Patos, $P B$, sobre $o$ atendimento imediato às vítimas de traumatismo dentário. Materiais e método: foi realizado um estudo transversal com 138 professores, em 36 escolas cadastradas no Instituto Nacional de Estudos e Pesquisas Educacionais Anísio Teixeira. A coleta de dados foi realizada mediante a aplicação de um questionário estruturado acerca da conduta imediata com a criança após traumatismo dental. Os dados obtidos foram analisados a partir da estatística descritiva e inferencial bivariada (teste Qui-Quadrado). Resultados: a idade dos educadores variou de 19 a 60 anos, com maioria do gênero feminino (96,4\%). Quando perguntados sobre a experiência com primeiros socorros em vítima de trauma dental, apenas $16,7 \%$ responderam que já tiveram algum tipo de experiência. Desses, $43,5 \%$ foi no atendimento à vítima. A maioria dos entrevistados $(85,1 \%)$ concordou que, em todos os casos de trauma, o cirurgião-dentista precisa ser consultado, entretanto, apenas 36,9\% da amostra acredita que apenas o trauma com deslocamento total do dente do alvéolo traz repercussões desfavoráveis. Frente à avulsão, 44,2\% dos professores escovariam o dente e posteriormente procurariam o cirurgião-dentista, e $77,5 \%$ dos participantes acreditam que o tempo para $o$ atendimento ao paciente vítima de avulsão deve ser o mais rápido possível. Conclusão: os resultados sugerem que a maioria dos professores entrevistados não tem conhecimento suficiente sobre como proceder em casos de trauma dentário, por não ter recebido esse treinamento ou por ter recebido pouca informação sobre o assunto.

Palavras-chave: Avulsão dentária. Docentes. Traumatologia.

\section{Introdução}

O traumatismo orofacial, que acomete o complexo dentoalveolar e os tecidos moles orais, consiste em um relevante problema de saúde pública ${ }^{1}$, de-

Cirurgiã-dentista, Universidade Federal de Campina Grande, Patos, PB, Brasil.

Graduando do curso de Odontologia, Universidade Federal de Campina Grande, Patos, PB, Brasil.

Mestra em Diagnóstico Bucal, professora da Faculdade de Odontologia da Universidade Federal de Campina Grande, Patos, PB, Brasil.

Doutora em Endodontia, professora da Faculdade de Odontologia da Universidade Federal de Campina Grande, Patos, PB, Brasil.

Doutora em Odontologia, professora da Faculdade de Odontologia da Universidade Federal de Campina Grande, Patos, PB, Brasil. 
vido à elevada prevalência, pela abrangência de indivíduos acometidos em faixas etárias, localidades ou ambientes variados e devido à possibilidade de danos estéticos, funcionais e emocionais, tanto aos pacientes como a seus familiares ${ }^{2}$.

As lesões traumáticas aos dentes são comuns nos jovens, principalmente naqueles que são vítimas de acidentes automobilísticos, jogos e esportes em geral ${ }^{3}$. O trauma dental pode variar de uma pequena fratura de esmalte até um extenso dano maxilofacial, envolvendo as estruturas de apoio e/ ou deslocamento dos dentes ${ }^{4}$.

Muitos dentes traumatizados são perdidos ou apresentam um prognóstico sombrio devido à falta de informação da população quanto às medidas de pronto atendimento adequadas no momento do acidente. A resistência ao uso dos equipamentos de segurança tem-se revelado uma constante característica dos brasileiros ${ }^{5}$.

Tendo em vista que a criança passa grande parte do tempo na escola e que as atividades esportivas são fatores predisponentes ao traumatismo, um estudo prévio mostrou a forte necessidade da inclusão do conhecimento de urgência no currículo dos professores escolares ${ }^{6}$, que são multiplicadores de informação, havendo, dessa forma, interferência positiva na promoção da saúde e na prevenção das complicações mais severas ${ }^{7}$.

Um dos fatores que podem determinar baixos índices de procura por atendimento odontológico é o fato de os reflexos do traumatismo dentário serem pouco conhecidos pela população. Em se tratando de trauma de baixa severidade e, aparentemente, pouco comprometimento dentário, pode-se, muitas vezes, evidenciar a ausência de procura por esse tipo de atendimento. Porém, deve-se salientar que, além da importância do tratamento dos dentes traumatizados em função de seu alto impacto na qualidade de vida dos indivíduos, há ainda a possibilidade de ocorrência de outras manifestações secundárias em longo prazo, como a reabsorção dentária ${ }^{8}$.

Diante da importância de se preparar os professores de crianças e adolescentes para a realização dos procedimentos corretos diante de um quadro de traumatismo dentário, torna-se necessário a priori conhecer a realidade desses profissionais, para que seja possível averiguar as maiores dificuldades e limitações de informações adquiridas sobre o tema, para que por meio de trabalhos científicos seja possível direcionar esse aprendizado.

Assim sendo, o presente estudo teve como objetivo avaliar o nível de conhecimento dos professores do ensino fundamental I da rede particular de ensino na cidade de Patos, $\mathrm{PB}$, sobre o atendimento imediato de vítimas de traumatismo dentário em ambiente escolar, a fim de auxiliar as estratégias de educação em saúde no fornecimento de informações a esses profissionais.

\section{Materiais e método}

O presente trabalho trata-se de um estudo transversal do tipo censitário, realizado com 138 educadores das 36 escolas da rede particular de ensino do município de Patos, PB. A coleta dos dados foi realizada por meio de um questionário estruturado contendo nove perguntas, elaboradas pelos próprios pesquisadores do trabalho, com dados referentes à formação acadêmica, experiências prévias sobre o assunto abordado e questões sobre o atendimento imediato de crianças vítimas de traumatismo dental em ambiente escolar. $O$ período de coleta e análise dos dados dos participantes foi entre os meses de outubro de 2013 a abril de 2014.

De acordo com a Resolução 466/2012 do Conselho Nacional de Saúde, este trabalho foi aprovado pelo comitê de ética em pesquisa (CAAE 17214613.1.0000.5181) e segue os padrões exigidos pela Declaração de Helsinque. Previamente à coleta dos dados, foi explicado aos participantes o objetivo da pesquisa e solicitada sua participação após a assinatura do termo de consentimento livre e esclarecido.

A aplicação dos questionários foi feita nas escolas em que os professores exercem suas atividades, em um momento que fosse conveniente para eles, na tentativa de interferir minimamente na rotina de trabalho dos profissionais e dos alunos. A autenticidade das respostas foi avaliada por meio do método de face, em que $10 \%$ dos entrevistados explicaram as perguntas com suas próprias palavras, o que permite concluir que o instrumento foi bem compreendido pelos participantes ${ }^{9}$.

Utilizou-se como critério de inclusão todos os professores do ensino fundamental I da rede particular de ensino da cidade de Patos, PB, que estavam regularmente trabalhando nas escolas registradas pelo Instituto Nacional de Estudos e Pesquisas Educacionais Anísio Teixeira e que assinaram o termo de consentimento livre e esclarecido.

Os dados obtidos neste estudo foram tabulados no programa Statistical Package for Social Sciences, versão 18.0, e são apresentados em formato de tabela, com apresentação de valores absolutos e percentuais. Para a análise estatística dos dados, foi utilizado o teste Qui-Quadrado (nível de significância de 5\%).

\section{Resultados}

Da amostra total, participaram do estudo 138 educadores, de 36 escolas pertencentes ao município. Foram excluídas da pesquisa uma instituição de ensino cuja direção recusou a participação no estudo e outra que havia fechado. A idade dos educadores variou entre 19 e 60 anos, com média de idade de 32 anos $( \pm 7,99)$, com predominância de indivíduos do sexo feminino $(96,4 \%, \mathrm{n}=133)$. A 
maioria dos participantes leciona em turmas do $1^{\circ}$ ano $(25,4 \%)$, como mostra a Tabela 1 .

Perguntados sobre a experiência com primeiros socorros à vítima de trauma dental, apenas $16,7 \%$ relataram ter algum tipo de experiência. Desses, um grupo de 43,5\% teve experiência no atendimento à vítima (Tabela 1 ).

Tabela 1 - Caracterização da amostra

\begin{tabular}{l|r|r}
\multicolumn{1}{c|}{ Variáveis } & \multicolumn{1}{c}{$\mathrm{N}$} & \multicolumn{1}{c}{$\%$} \\
\hline Sexo & 5 & 3,6 \\
Masculino & 133 & 96,4 \\
Feminino & & \\
Turma que ministra aulas & 35 & 25,4 \\
$1^{\circ}$ ano & 27 & 19,6 \\
$2^{\circ}$ ano & 25 & 18,1 \\
$3^{\circ}$ ano & 30 & 21,7 \\
$4^{\circ}$ ano & 21 & 15,2 \\
$5^{\circ}$ ano & & \\
Experiência com vítimas de trauma dental & 23 & 16,7 \\
Sim & 115 & 83,3 \\
Não & & \\
Tipo de experiência & 60 & 43,5 \\
Atendimento & 30 & 21,7 \\
Palestra & 36 & 26,1 \\
Leitura & 12 & 8,7 \\
Outros & & \\
\hline Fonte: todas as tabelas são de elaboração dos autores com base nos dados
\end{tabular}

da pesquisa.
No caso da orientação de uma vítima de trauma dentário, a maioria dos entrevistados $(85,1 \%)$ julgou necessário procurar um cirurgião-dentista, independente do trauma ocorrido, enquanto que 13,4\% dos educadores julgaram necessário procurar um profissional cirurgião-dentista apenas em casos mais graves (Tabela 2).

Quando indagados sobre qual tipo de trauma teria repercussão desfavorável no meio bucal, $36,9 \%$ afirmaram que seriam apenas os casos de deslocamento total do elemento dentário, 33,6\% responderam que o deslocamento parcial do dente pode ter repercussão desfavorável, e 29,5\% responderam que apenas o trauma, sem deslocamento dentário, já pode apresentar repercussão desfavorável (Tabela 2 ).

Ao se perguntar sobre o modo de agir e a melhor maneira de armazenar os elementos em casos de avulsão dentária, 44,2\% escovariam o dente deslocado antes de seguir para o dentista, outros $42,8 \%$ dos participantes armazenariam o dente em leite e encaminhariam para o profissional capacitado (Tabela 2).

Em relação ao tempo ideal para procurar atendimento em casos de dentes avulsionados, a maioria $(77,5 \%)$ respondeu que o tempo ideal seria imediatamente após o trauma, outros 20,3\% declararam não ser primordial o tempo para o atendimento, como apresentado na Tabela 2.

Tabela 2 - Conhecimento dos professores sobre o atendimento imediato à vítima de traumatismo

\begin{tabular}{|c|c|c|}
\hline Variáveis & $\mathrm{N}$ & $\%$ \\
\hline \multicolumn{3}{|l|}{ Conduta de orientação à vítima } \\
\hline Sempre orienta & 117 & 85,1 \\
\hline Às vezes orienta & 19 & 13,4 \\
\hline Não orienta & 2 & 1,5 \\
\hline \multicolumn{3}{|c|}{ Tipo de trauma que pode causar repercussão desfavorável* } \\
\hline Total & 80 & 36,9 \\
\hline Parcial & 73 & 33,6 \\
\hline Ausência de deslocamento & 64 & 29,5 \\
\hline \multicolumn{3}{|c|}{ Reação dos professores em caso de deslocamento de um dente para fora da boca } \\
\hline Reimplante & 12 & 8,7 \\
\hline Limpar & 61 & 44,2 \\
\hline Armazenar & 59 & 42,8 \\
\hline Não respondeu & 6 & 4,3 \\
\hline \multicolumn{3}{|c|}{ Tempo para o atendimento após deslocamento total de um dente } \\
\hline O mais rápido & 107 & 77,5 \\
\hline Não importa & 28 & 20,3 \\
\hline Não respondeu & 3 & 2,2 \\
\hline
\end{tabular}

* Os participantes poderiam responder a mais de uma alternativa.

Ao se avaliar a influência da variável sexo dos participantes do estudo em relação à experiência de traumatismo dentário quanto à experiência em primeiros socorros à vítima, ao tempo que um dente pode ficar fora do alvéolo, à conduta diante da avulsão dentária e à orientação em procurar um cirurgião-dentista, em relação à gravidade do caso, não se verificou nenhuma associação estatística entre as variáveis analisadas (Tabela 3 ). 
Tabela 3 - Análise bivariada da experiência com o traumatismo dentário e a variável sexo dos participantes do estudo

\begin{tabular}{|c|c|c|c|c|}
\hline \multirow[b]{2}{*}{ Variáveis } & \multicolumn{4}{|c|}{ Sexo } \\
\hline & $\begin{array}{c}\text { Masculino } \\
\mathrm{n}(\%)\end{array}$ & $\begin{array}{c}\text { Feminino } \\
\mathrm{n}(\%)\end{array}$ & $\begin{array}{l}\text { Total } \\
\mathrm{n}(\%)\end{array}$ & $\begin{array}{l}\text { Valor } \\
\text { de } \mathrm{p}^{(1)}\end{array}$ \\
\hline \multicolumn{5}{|c|}{ Experiência com primeiros socorros à vítima de trauma dental } \\
\hline Sim & $1(4,3)$ & $22(95,7)$ & 23 & \multirow{2}{*}{0,604} \\
\hline Não & $4(3,5)$ & $111(96,5)$ & 115 & \\
\hline \multicolumn{5}{|c|}{ Tempo que um dente pode ficar fora do alvéolo } \\
\hline Não importa & $1(3,6)$ & $27(96,4)$ & 28 & \multirow{2}{*}{0,723} \\
\hline O mínimo possível & $4(3,7)$ & $103(96,3)$ & 107 & \\
\hline \multicolumn{5}{|l|}{ Conduta diante da avulsão dentária } \\
\hline Reimplantar & $0(100,0)$ & $12(0,0)$ & 12 & \multirow{3}{*}{0,203} \\
\hline Limpar & $3(4,9)$ & $58(95,1)$ & 61 & \\
\hline Armazenar & $2(3,4)$ & $57(96,6)$ & 59 & \\
\hline \multicolumn{5}{|c|}{ Orientação para procurar o dentista dependendo da gravidade do trauma } \\
\hline Sempre orienta & $3(2,6)$ & $111(97,4)$ & 114 & \multirow{3}{*}{0,723} \\
\hline Às vezes orienta & $2(11,1)$ & $16(88,9)$ & 18 & \\
\hline Nunca orienta & $0(0,0)$ & $2(100,0)$ & 2 & \\
\hline
\end{tabular}

* Estatisticamente significante $(p<0,05)$.

(1) Teste Qui-Quadrado de Pearson.

De maneira semelhante, ao se investigar a influência da idade dos participantes em relação à experiência com traumatismo dentário e primeiros socorros à vítima, quanto ao tempo que um dente pode ficar fora do alvéolo, à conduta diante da avulsão dentária e à orientação em procurar o cirurgião-dentista, em relação à gravidade do caso, verificou-se uma associação estatisticamente significativa quanto à experiência em primeiros socorros à vítima $(p=0,003)$, revelando que os participantes mais jovens são aqueles que têm maior experiência (Tabela 4).

Tabela 4 - Análise bivariada da experiência com traumatismo dentário e a idade dos participantes do estudo

\begin{tabular}{|c|c|c|c|c|c|}
\hline \multirow[b]{2}{*}{ Variáveis } & \multicolumn{5}{|c|}{ Faixa etária } \\
\hline & $\begin{array}{c}19-32 \text { anos } \\
\mathrm{n}(\%)\end{array}$ & $\begin{array}{c}33-46 \text { anos } \\
n(\%)\end{array}$ & $\begin{array}{c}47-60 \text { anos } \\
n(\%)\end{array}$ & $\begin{array}{c}\text { Total } \\
\mathrm{n}\end{array}$ & $\begin{array}{l}\text { Valor de } \\
\qquad p^{(1)}\end{array}$ \\
\hline \multicolumn{6}{|c|}{$\begin{array}{l}\text { Experiência com primeiros socorros à vítima de } \\
\text { trauma dental }\end{array}$} \\
\hline Sim & $12(52,2)$ & $7(30,4)$ & $4(17,4)$ & 23 & \multirow{2}{*}{$0,003^{*}$} \\
\hline Não & $61(53,0)$ & $52(45,2)$ & $2(1,7)$ & 115 & \\
\hline \multicolumn{6}{|c|}{ Tempo que um dente pode ficar fora do alvéolo } \\
\hline Não importa & $15(53,6)$ & $12(42,9)$ & $1(3,6)$ & 28 & \multirow{2}{*}{0,058} \\
\hline O mínimo possível & $56(52,3)$ & $46(43,0)$ & $5(4,7)$ & 107 & \\
\hline \multicolumn{6}{|l|}{ Conduta diante da avulsão dentária } \\
\hline Reimplantar & $7(58,3)$ & $5(41,7)$ & $0(0,0)$ & 12 & \multirow{3}{*}{0,604} \\
\hline Limpar & $33(54,1)$ & $24(39,3)$ & $4(6,6)$ & 61 & \\
\hline Armazenar & $31(52,5)$ & $27(45,8)$ & $1(1,7)$ & 59 & \\
\hline \multicolumn{6}{|c|}{$\begin{array}{l}\text { Orientação em procurar o dentista dependendo da } \\
\text { gravidade do trauma }\end{array}$} \\
\hline Sempre orienta & $60(52,6)$ & $51(44,7)$ & $3(2,6)$ & 114 & \multirow{3}{*}{0,967} \\
\hline Às vezes orienta & $9(50,0)$ & $6(33,3)$ & $3(16,7)$ & 18 & \\
\hline Nunca orienta & $2(100,0)$ & $0(0,0)$ & $0(0,0)$ & 2 & \\
\hline
\end{tabular}

${ }^{*}$ Estatisticamente significante $(\mathrm{p}<0,05)$.

(1) Teste Qui-Quadrado de Pearson.

\section{Discussão}

No presente estudo, verificou-se homogeneidade na maioria das respostas, o que comprovou o entendimento das questões que compõem o questionário. Diante dos dados obtidos, sugere-se a clara falta de conhecimento dos professores sobre primeiros socorros em casos de traumatismo dentário, mostrando assim uma deficiência de conhecimento sobre o assunto, corroborando os dados de $\operatorname{Lima}^{10}$ (2010), em que apenas $7,1 \%$, de um total de 212 educadores, apresentaram conhecimento sobre trauma dental. 
De maneira semelhante, os dados aqui obtidos concordam com o trabalho de Granville-Garcia et al. ${ }^{11}$ (2007), que entrevistaram 79 professores de educação física da cidade de Caruaru, PE, e obtiveram um percentual de $20,3 \%$ de participantes que sabiam definir trauma dental, revelando pouco conhecimento sobre o tema. Assim, foi possível verificar que esses profissionais não estão capacitados para um correto atendimento logo após um trauma dental.

De modo congruente a este trabalho, o estudo de $\operatorname{Costa}^{12}$ (2004), avaliando o conhecimento e as atitudes de uma amostra de 177 professores do município de São Paulo, SP, verificou que a minoria dos professores tivera alguma experiência com trauma dental ( $23,2 \%$ dos entrevistados).

É imperioso ressaltar a importância de se levar o aluno vítima de trauma imediatamente ao cirurgião-dentista, e de maneira bastante positiva a maioria dos educadores participantes deste estudo demonstraram ter essa consciência. Esse achado é semelhante ao obtido por Lima ${ }^{10}$ (2010), em que a minoria $(15,6 \%)$ levaria o aluno a um cirurgião-dentista apenas em caso de avulsão, e 1,5\% relatou não ser necessária essa orientação. Mesmo assim, ainda há a necessidade de se orientar mais esses profissionais formadores de opinião, para que todos tenham o conhecimento correto, a fim de minimizar os danos às vítimas de trauma.

Lacunas no conhecimento científico da maioria dos professores foram observadas, pois muitos apresentaram respostas incoerentes e que não correspondiam com o indicado para o caso, como quando indagados sobre o tipo de trauma possível de causar repercussões desfavoráveis, a reação em caso de deslocamento dentário e o tempo para atendimento após o deslocamento do dente. Esses achados são concordantes com o estudo de Cordeiro et al. ${ }^{13}$ (2010), em que foram entrevistadas 58 voluntárias, entre diretoras, professoras e berçaristas de creches públicas de referência em atenção a crianças provenientes de famílias com situação socioeconômica vulnerável em Campina Grande, PB. Quando perguntados quanto aos cuidados com o dente avulsionado, se o dente fosse encontrado, ele seria lavado com água (37,9\%) e armazenado em algodão seco (20,7\%).

Além das condições de armazenamento do elemento dentário avulsionado, deve-se atentar para o tempo entre $o$ acidente e $o$ atendimento pelo profissional cirurgião-dentista, o que determina o prognóstico e as alternativas a serem seguidas no plano de tratamento ${ }^{8}$.

Nota-se, conforme o estudo de Berti ${ }^{14}$ (2011), que a própria boca é um dos locais mais preconizados para o armazenamento, devido às propriedades da saliva na manutenção das condições favoráveis para as células do ligamento periodontal quando lesionadas. Tendo o alvéolo como melhor meio de armazenamento, também podem ser usados, em ordem de preferência, a própria saliva, o leite e a solução fisiológica.
Os resultados encontrados no presente estudo sugerem que a maioria dos professores entrevistados não tem conhecimento suficiente sobre como proceder em casos de trauma dentário, por não ter recebido esse treinamento ou por ter recebido pouca informação sobre o assunto. De modo similar, Barbosa $^{15}$ (2012), em pesquisa realizada com noventa professores atuantes em escolas públicas da cidade de Piracicaba, SP, documentou que a maioria deles nunca participou de um curso de primeiros socorros (64\%) ou nunca recebeu qualquer informação sobre traumatismo dental (63\%). Dos $37 \%$ que disseram já ter recebido algum tipo de informação sobre trauma dental, a maioria obteve tais informações por meio de cursos e palestras (12,23\%). Esses percentuais reafirmam a pressuposição de que o conhecimento sobre traumatismo dental ainda é precário entre os profissionais da educação básica.

Percebe-se que os primeiros socorros não são um componente fundamental na formação do professor, visto que já foi discutida a sua grande importância. A falta de informação pode, portanto, levar a um comprometimento de prognóstico nos casos de reimplante dentário. Os resultados, quanto à importância do tempo para o sucesso do tratamento concordam com a afirmação de Costa et al. ${ }^{16}$ (2004), em que o prognóstico é favorável se o reimplante for realizado em até 30 minutos depois do acidente e que o tempo influencia na presença ou não de reabsorções radiculares. Em pesquisa realizada por $\operatorname{Berti}^{14}$ (2011), dos 76 entrevistados, 81,57\% teriam a iniciativa de conduzir a vítima ao dentista imediatamente após o trauma, resultado compatível com o do presente estudo, e esse é um fator importante para a definição de um tratamento e de um possível prognóstico do caso.

Foi possível constatar, neste estudo, que, ao ser avaliada a influência das variáveis sexo e idade dos participantes, sobre a experiência com traumatismo dentário, há uma associação estatisticamente significativa na relação entre a idade e a experiência com os primeiros socorros à vítima de trauma, uma vez que as pessoas mais jovens revelaram ter mais contato com a temática.

Esse contato pode ter acontecido de diversas maneiras, ou com a prestação de primeiros socorros propriamente dita, ou com leituras sobre o tema, bem como a vivência em palestras abordando as melhores condutas. Assim, pode-se supor que as pessoas mais jovens valorizam mais o assunto e buscam cada vez mais oferecer o melhor para o paciente traumatizado. Nesse contexto, sugere-se que cursos de capacitações sejam desenvolvidos, promovendo amplo acesso a todos os profissionais da área que desejem se atualizar, a fim de favorecer o melhor prognóstico, principalmente, em casos mais severos.

Pôde-se perceber que, por não ter recebido qualquer informação sobre o assunto, a maioria dos entrevistados teve dificuldade em responder aos questionários, o que ficou demonstrado na pouca 
qualidade das respostas. Propõe-se medidas educativas e produção de material educativo voltados para a capacitação desses educadores, assim como, posteriormente, a realização de futuros estudos que possam acompanhar a evolução do conhecimento dos educadores, detectando sempre a ocorrência de dificuldades por parte desses profissionais.

\section{Conclusão}

Os professores entrevistados demonstram pouco conhecimento sobre tratamento de urgência em casos de traumas dentários, indicando grande necessidade de capacitação e acesso à informação, para que possa ser feito o manejo inicial adequado do traumatizado, evitando assim o comprometimento do prognóstico e colaborando para o sucesso do tratamento das lesões.

\section{Abstract}

Objective: To identify the level of knowledge of private elementary school teachers from the city of Patos, PB, Brazil on the immediate assistance of dental trauma victims. Methods: Cross-sectional study with 138 teachers in 36 schools registered at the National Institute of Educational Studies and Research Anísio Teixeira. Data collection was performed by applying a structured questionnaire about immediate child assistance after dental trauma. Data obtained were analyzed using descriptive and bivariate inferential statistics (chi-square test). Results: The age of teachers ranged from 19 to 60 years and most of them were women (96.4\%). When asked about experience with first aid to dental trauma victims, only $16.7 \%$ said they already had some experience, wherein $43.5 \%$ had it by assisting the victims. Most respondents (85.1\%) agree that in all cases of trauma, the dentist needs to be consulted, but only $36.9 \%$ of the sample believes that the trauma with full alveolar tooth displacement alone brings unfavorable repercussions. Facing avulsion, $44.2 \%$ of teachers would brush the tooth and then seek the dentist, and $77.5 \%$ of respondents believe that the time for assisting a patient victim of avulsion should be as soon as possible. Conclusions: The results suggest that most of the teachers interviewed do not have enough knowledge on how to proceed in cases of dental trauma because they have not received this type of training or have received little information about it.

Keywords: Tooth Avulsion. Teachers. Traumatology.

\section{Referências}

1. Glendor U. Aetiology and risk factors related to traumatic dental injuries - a review of literature. Dent Traumatol 2009; 25(1):19-31.

2. Colak I, Markovic D, Petrovic B, Peric T, Milenkovic A. A retrospective study of intrusive injuries in primary dentition. Dent Traumatol 2009; 25(6):605-10.

3. Rezende FMC, Gaujac C, Rocha AC, Peres MPSM. A prospective study of dentoalveolar trauma at the Hospital das
Clínicas, São Paulo University Medical School. Clinics 2007; 62(2):133-8.

4. Chan AW, Wong TK, Cheung GS. Lay knowledge of physical health education teachers about the emergency management of dental trauma. Dent Traumatol 2001; 17(2):77-85.

5. Ferrari CH, Medeiros JMF. Dental trauma and level of information: mouthguard use in different contacts sports. Dent Traumatol 2002; 18(3):144-7.

6. Al-jundi SH. Type of treatment, prognosis, and estimation of time spent to manage dental trauma in late presentation cases at a dental teaching hospital: a longitudinal and retrospective study. Dent Traumatol 2004; 20(1):1-5.

7. Hanan AS, Costa SK. Conhecimento dos professores de $1^{a}$ a $4^{a}$ série de escolas públicas municipais de Manaus/AM frente à avulsão dentária. Pesq Bras Odontoped Clin Integr 2010; 10(11):27-33.

8. Cortes MIS, Marcenes W, Sheiham A. Impact of traumatic injuries to the permanent teeth on oral health related quality of life of 12-14 year old in Brazilian schoolchildren. Community Dent Oral Epidemiol, Denmark 2002; 30(3):193-8.

9. Frankfort-Nachmias C, Nachmias D. Research methods in the social sciences. London: Edward Arnold; 1992.

10. Lima DC. Traumatismo alvéolo-dentário: prevalência em crianças e conhecimento de educadores do ensino fundamental [Tese de Doutorado]. Araçatuba: Faculdade de Odontologia de Araçatuba da Universidade Estadual de Paulista Júlio de Mesquita Filho; 2010. Disponível em URL: http://www.athena.biblioteca.unesp.br/exlibris/bd/ bfo/33004021074P1/2010/lima_dc_dr_araca.pdf.

11. Granville-Garciai AF, Limaii EM, Santosii PG, Menezesiii VA. Avaliação do conhecimento dos professores de educação física de Caruaru-PE sobre avulsão-reimplante. Pesq Bras Odontoped Clin Integr 2007; 7(1):15-20.

12. Costa ABM. Traumatismos alvéolo-dentários: avaliação dos conhecimentos e atitudes de uma amostra de professores do ensino fundamental do município de São Paulo [Dissertação de Mestrado]. São Paulo: Universidade de São Paulo, São Paulo; 2004. Disponível em URL: http://www.teses.usp.br/ teses/disponiveis/23/23143/tde-02022005-130347/pt-br.php.

13. Cordeiro PM, Fontes LBC, Granville-Garcia AF, Maciel MAS, Lucas RSCC. Perception of the directors, professors and nursery school professionals of public day-care centers on the orofacial trauma. Rev Odontol Unesp 2010; 39(3):169-73.

14. Berti M, Furlanetto DLC, Refosco MZ. Avaliação do conhecimento de professores do ensino fundamental sobre o tema avulsão dentária. Pesq Bras Odontoped Clin Integr 2011; 11(3):381-6.

15. Barbosa MTN. Conhecimento dos professores sobre traumatismo dentário em escolas públicas de Piracicaba, SP. [Trabalho de conclusão de curso]. São Paulo: Faculdade de Odontologia de Piracicaba; 2012. Disponível em URL: http://www. bibliotecadigital.unicamp.br/document/?code $=000909437$.

16. Costa AJM, Lasserre FS, Westphalen VPD, Deonizio MDA, Silva Neto UX, Sousa MH. Reimplante dentário tardio: relato de caso clínico. Rev Clin Pesq Odontol 2004; 1(2):41-3.

Endereço para correspondência:

Tassia Cristina de Almeida Pinto-Sarmento Rua Antônio de Sousa Lopes, 120, apto. 1.002 - Catolé

58410-180 Campina Grande, PB

Telefone: (83) 98858-2771

E-mail: tassiapinto@yahoo.com.br

Recebido: 13/02/15. Aceito: 01/12/15. 